Review

\title{
Eukaryotic LYR Proteins Interact with Mitochondrial Protein Complexes
}

\section{Heike Angerer}

Goethe University Frankfurt, Medical School, Institute of Biochemistry II, Structural Bioenergetics Group, Max-von-Laue Street 9, Frankfurt am Main 60438, Germany; E-Mail: Angerer@em.uni-frankfurt.de; Tel.: +49-69-798-29575

Academic Editor: Thorsten Berg

Received: 12 December 2014 / Accepted: 4 February 2015 / Published: 12 February 2015

\begin{abstract}
In eukaryotic cells, mitochondria host ancient essential bioenergetic and biosynthetic pathways. LYR (leucine/tyrosine/arginine) motif proteins (LYRMs) of the Complex1_LYR-like superfamily interact with protein complexes of bacterial origin. Many LYR proteins function as extra subunits (LYRM3 and LYRM6) or novel assembly factors (LYRM7, LYRM8, ACN9 and FMC1) of the oxidative phosphorylation (OXPHOS) core complexes. Structural insights into complex I accessory subunits LYRM6 and LYRM3 have been provided by analyses of EM and X-ray structures of complex I from bovine and the yeast Yarrowia lipolytica, respectively. Combined structural and biochemical studies revealed that LYRM6 resides at the matrix arm close to the ubiquinone reduction site. For LYRM3, a position at the distal proton-pumping membrane arm facing the matrix space is suggested. Both LYRMs are supposed to anchor an acyl-carrier protein (ACPM) independently to complex I. The function of this duplicated protein interaction of ACPM with respiratory complex I is still unknown. Analysis of protein-protein interaction screens, genetic analyses and predicted multi-domain LYRMs offer further clues on an interaction network and adaptor-like function of LYR proteins in mitochondria.
\end{abstract}

Keywords: LYR proteins; LYRM; LYR motif; mitochondria; OXPHOS complexes; respiratory complex I; mitochondrial acyl-carrier protein; ACPM; mitochondrial fatty acid synthesis type II; lipoic acid (6,8-dithio-octanoic acid); reactive oxygen species; insulin resistance 


\section{The LYR Motif}

The LYR proteins (LYRMs) of the Complex1_LYR-like superfamily are exclusively found in eukaryotes. The Complex1_LYR-like superfamily (Pfam clan CL0491) was manually curated by P. Coggill from the Wellcome Trust Sanger Institute/European Bioinformatics Institute (EMBL-EBI) [1], and it has three Pfam-A members: the LYR-, LYR_1- and LYR_2-protein family. LYRMs are basic $\sim 15 \mathrm{kDa}$ polypeptides and carry a conserved tripeptide L-Y-R (leucine/tyrosine/arginine) sequence close to the N-terminus (Table 1). Furthermore, some basic arginine/lysine residues and a highly-conserved phenylalanine are downstream elements of the LYR motif in the LYR- and LYR_1-protein family, respectively. The LYR_2 motif is characterized by the N-terminal L-Y-R sequence and a pattern of tyrosines/glycine at the C-terminus (reviewed in [2]). The human genome contains at least ten LYRMs that were predominantly identified as mitochondrial proteins (Figure 1) [3-6]; however, some members of this protein superfamily were also found in the cytosol or nucleus [7,8]. Human LYRMs are linked with diseases, such as insulin-resistance (LYRM1 [9]), muscular hypotonia (LYRM3 [10]), deficiency of multiple OXPHOS complexes (LYRM4 [11]), apoptosis in HIV-1 infection (LYRM6 [12]), encephalopathy and lactic acidosis (LYRM7/MZM1L [13]), infantile leukoencephalopathy (LYRM8/succinate dehydrogenase assembly factor (SDHAF) 1 [14]) and alcohol dependence (acetate non-utilizing protein (ACN) 9/SDHAF3 [15]). In this review, the human nomenclature of proteins is used throughout; otherwise, the nomenclature of the respective organism is indicated.

The original classification of LYRMs was based on LYRM4, which interacts with the cysteine desulfurase NFS1, a central component of the ancient Fe-S cluster biogenesis machinery [16,17]. Accordingly, there was an initial bias to assume a role in mitochondrial Fe-S cluster biogenesis for all LYRMs, e.g., prompted by the Pfam database (www.pfam.xpfam.org/clan/CL0491). However, LYRM4 seems to represent the only LYR protein that is directly involved in the first steps of Fe-S cluster generation. Furthermore, other LYRMs have been identified as accessory subunits or assembly factors of mitochondrial OXPHOS complexes I, II, III and V, respectively, and to play a role in acetate metabolism [2]. Nevertheless, a common specific physiological role of LYRMs and the structural role of the LYR motif are still unknown.

The tripeptide sequence L-Y-R is a central part of the LYR motif in LYRMs, but the entire motif requires further conserved amino acid residues (Table 1). However, it should be noted that the term "LYR proteins" has been used by some authors to classify proteins with an L-Y-R tripeptide, but lacking other features of the LYR motif. The sequence stretch L-Y-R is frequently found in mitochondrial proteins, e.g., in the sequence of complex I assembly factor NDUFAF3, complex II subunit SDHB, complex IV subunits 4 and 7A, complex V subunit B1 and ribosomal proteins (Supplemental Figure S1); however, these proteins are not LYRMs. Recently, the L-Y-R sequence was suggested to present an interaction site for mitochondrial co-chaperone binding [18]. The DNAJ-type III co-chaperone HSC20 was described as a component of the ISCU/HSPA9 protein complex that transfers Fe-S clusters from the scaffold protein ISCU to target proteins [19,20]. Complex II assembly factor LYRM8/SDHAF1, complex II subunit SDHB and complex III assembly factor LYRM7/MZM1L carry L-Y-R(-like) sequences (Table 1, Supplemental Figure S1). In co-immunoprecipitation and yeast two hybrid experiments, LYRM7, LYRM8 and SDHB were shown to interact with the co-chaperone HSC20 via the L-Y-R(-like) sequences $[18,20]$. However, subunit SDHB is not defined as LYRM, as analyzed using the bioinformatics tool Pfam search (www.pfam.xfam.org/search). 
Table 1. Human LYRMs in mitochondria. The theoretical molecular masses of LYRMs range between $10 \mathrm{kDa}$ and $22 \mathrm{kDa}$ and the theoretical pI between nine and 11. LYRMs are predominantly mitochondrial proteins; however, some LYRMs reside also in the cytoplasm or nucleus, as reported for LYRM1 and LYRM4, respectively [7,8]. The most important conserved residues of LYRMs are shown in the sequence alignment of the human proteins (highlighted in red). C, complex; ISC, iron sulfur cluster; COPP, complex I phylogenetic profile; AF, assembly factor.

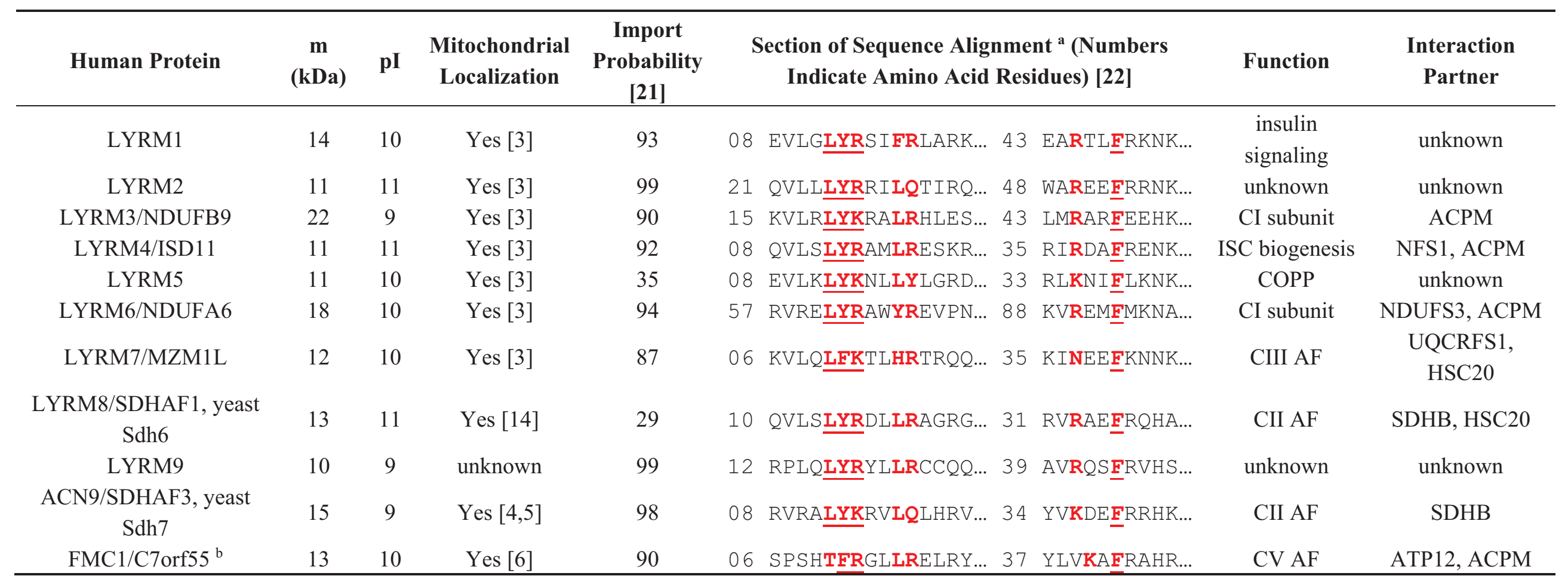

${ }^{a}$ ClustalW2, default parameters; the sequence of LYRM6 was manually shifted; ${ }^{b}$ formation of mitochondrial complexes protein 1 , not a LYRM, as analyzed using the Pfam search tool (see the main text). 


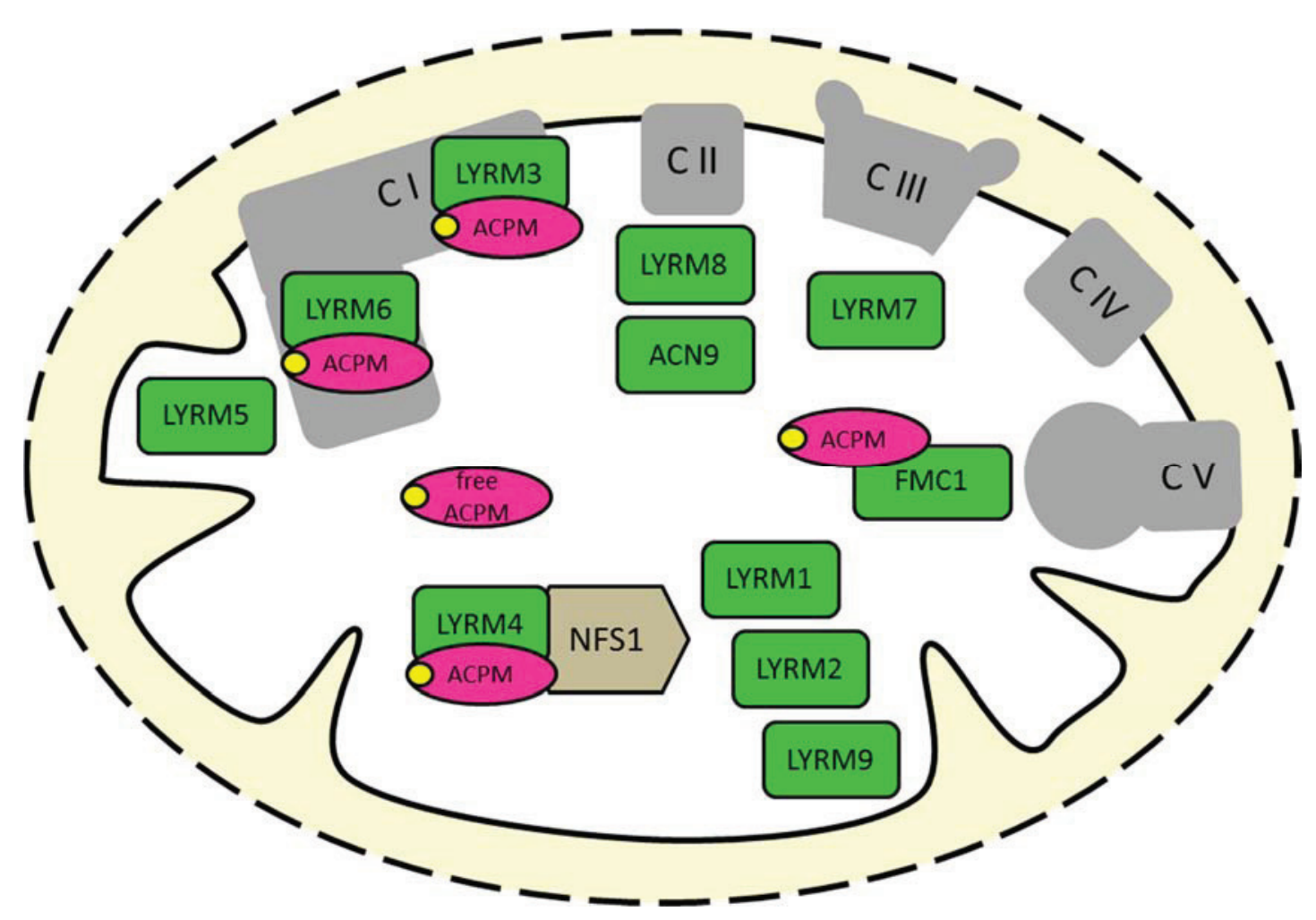

Figure 1. LYRMs in mitochondria of (higher) eukaryotes. Many LYRMs (green) are subunits or assembly factors of OXPHOS complexes. Four LYRMs interact with the mitochondrial acyl carrier protein (ACPM/NDUFAB1, magenta), which is also a duplicated subunit of complex I $[23,24]$. LYRM4 is an essential protein of the Fe-S cluster biogenesis machinery. LYRM5 is a complex I-related protein (see the main text). The function of LYRM1, LYRM2 and LYRM9 in mitochondria is unknown. C, complex; NFS1, cysteine desulfurase; yellow circles, phosphopantetheine cofactor of ACPM.

The downstream phenylalanine of the LYR motif (Table 1) might play an important role in the function of LYRMs. Complex I accessory subunit LYRM6/NDUFA6 (yeast NB4M) from the yeast Yarrowia lipolytica is essential for complex I activity and anchors a mitochondrial acyl-carrier protein ACPM/NDUFAB1- $\alpha$ (yeast ACPM1) to complex I [25] (Figure 2b). Mutation of the L-Y-R sequence of LYRM6 resulted in slightly reduced abundance of complex I in mitochondrial membranes with no effect on normalized complex I activity. A much more drastic effect was observed only after mutation of the L-Y-R sequence in conjunction with the conserved downstream phenylalanine (LYR $+F)$. Subunit ACPM1 was detached from the enzyme complex, and complex I activity was abolished. Thus, the LYR motif of subunit LYRM6 might be important to bind the ACPM subunit. Complexome profiling of intact mitochondria showed that LYRM6 co-exists as complex I-associated and in free form in the matrix space [25]. It cannot be excluded that the soluble matrix LYRM6 might have extra functions, e.g., as a maturation factor of the Fe-S cluster containing central subunits increasing the abundance of complex I in mitochondrial membranes. Taken together, LYRMs are basic hydrophilic proteins interacting with mitochondrial macromolecular complexes, such as the OXPHOS complexes and the NFS1/ISCU complex of Fe-S cluster biogenesis. There are indications that LYRMs are adaptor-like mitochondrial factors that promote the assembly and function of multi-subunit protein complexes. 

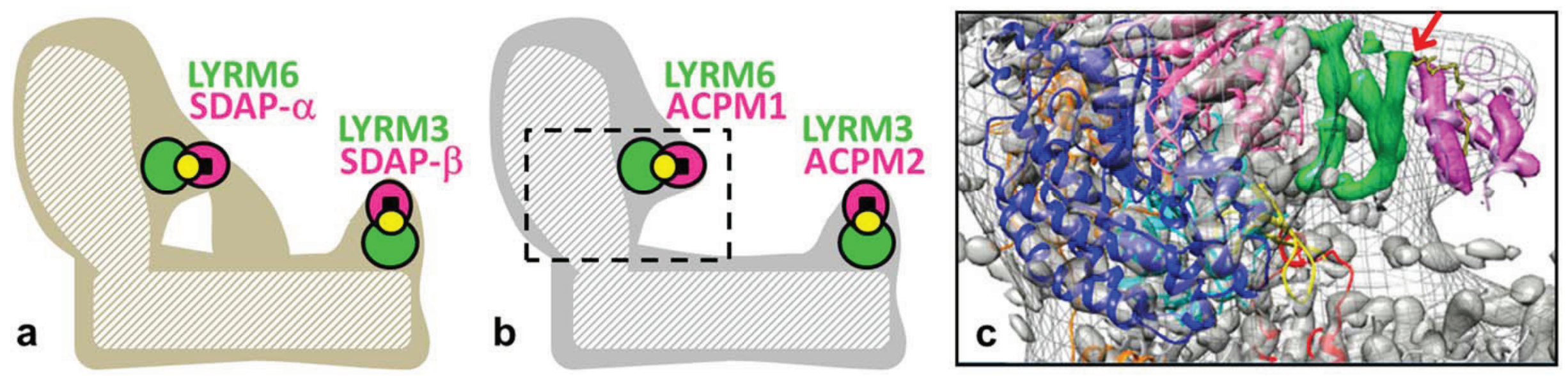

Figure 2. LYRM subunits of mitochondrial complex I. (a) Schematic model of complex I from Bos taurus after [24]. The dashed area represents the central subunits harboring the catalytic core functions. Accessory subunits (filled areas) form a scaffold around the central subunits. Two accessory domains, one at the peripheral matrix arm and one at the distal proton pumping membrane arm, are formed by LYRMs (green). LYRM6 and LYRM3 interact with the mitochondrial acyl-carrier protein subunit (ACPM, bovine SDAP, magenta) that carries phosphopantetheine cofactors (yellow circles) and, additionally, acyl groups (black squares). (b) Schematic model of Yarrowia lipolytica complex I in analogy to bovine complex I [23]. The frame in (b) indicates the zoom window of the structural modeling of subunits LYRM6 and ACPM1 in (c) [25]. (c) Overlay of X-ray electron density map (gray surface, contour level 1.9) with an EM envelope of Yarrowia lipolytica complex I (gray mesh) and the X-ray structure of bacterial Thermus thermophilus complex I (PDB file 4HEA); central subunits: NDUFS2 subunit (NQO4, blue; N-terminal $\beta$-sheet highlighted in yellow), NDUFS7 subunit (NQO6, cyan), NDUFS8 subunit (NQO9, orange), NDUFS3 subunit (NQO5, hot pink), loop connecting helices 1 and 2 of subunit ND3 (NQO7, red); structure of ACP from E. coli (PDB file 2FAE, pink; decanoyl phosphopantetheine, yellow stick representation) fitted to electron density (magenta); please compare the original figure of the publication [25]. Electron density features tentatively assigned to subunit LYRM6 are highlighted in green. The red arrow indicates the strong contact between LYRM6 and the modelled phosphate group of the phosphopantetheine cofactor from ACPM1. 


\section{The Diverse Roles of the LYR Motif Proteins in Mitochondria}

\subsection{Complex I Subunits LYRM3, LYRM6 and ACPM}

Mitochondrial complex I (NADH:ubiquinone oxidoreductase) is an L-shaped membrane protein with a hydrophilic peripheral arm and a hydrophobic membrane arm [26,27]. Interestingly, yeast and mammalian complex I contain two LYRMs, LYRM6/NDUFA6 (yeast NB4M, bovine B14) and LYRM3/NDUFB9 (yeast NI2M, bovine B22), and two versions/copies of the acyl-carrier protein subunit ACPM/NDUFAB1- $\alpha / \beta$ (yeast ACPM1/2, bovine SDAP- $\alpha / \beta$ ) $[24,28,29]$. The basic LYRM3 and LYRM6 (pI = 9 and 10) might bind the acidic ACPM (pI without phosphopantetheine cofactor $=4$ ) to complex I via strong polar interactions, forming essential accessory domains. Based on structural and biochemical characterization of complex I from the lyrm6 $\Delta$ deletion strain, the LYRM6/ACPM1 domain is located at the matrix arm close to the ubiquinone reduction site (Q module). In HIV-1 infected T-cells, the gene of LYRM6 is downregulated, and the activity of complex I is impaired, resulting in the induction of the apoptotic pathway [12]. The deletion studies in the model organism Yarrowia lipolytica suggested that the downregulation of human LYRM6 in HIV-infected T-cells leads to the loss of the LYRM6/ACPM- $\alpha$ domain of human complex I that is essential for catalytic activity and finally results in cell death. Subunit LYRM3 has been shown biochemically to reside at the distal part of the membrane

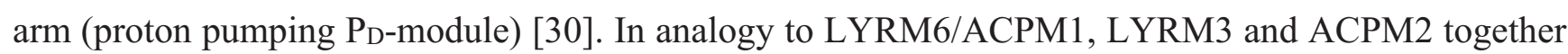
might form a common "duplicated" accessory domain of mitochondrial complex I (Figure 2). In fact, in the EM structure of bovine complex I, the SDAP- $\beta$ subunit (ACPM2) was identified at the tip of an accessory domain at the matrix-faced part of the $P_{D}$-module [24]. Thus, the accessory domain LYRM6/ACPM- $\alpha$ has been described for the yeast and bovine complex; and the LYRM3/ACPM- $\beta$ domain has been proposed for the bovine enzyme[23,24]. Deletion studies of ACPM1 and ACPM2 in Yarrowia lipolytica revealed that ACPM1 is an essential protein for the yeast, while ACPM2 is essential for complex I assembly/stability [31]. Downregulation of human ACPM compromises protein lipoylation, abolishes specific complex I activity and ultimately results in cell death [32]. ACPM is an essential protein of the mitochondrial fatty acid synthesis type II that forms octanoyl-ACPM precursors for the biosynthesis of lipoic acid (6,8-dithio-octanoic acid) [33] and of organelle-specific lipids [34]. In general, lipoylation of mitochondrial $\alpha$-keto dehydrogenases is indispensable for mitochondrial metabolism, and lipoic acid is not a vitamin that can be supplied externally $[32,33,35,36]$. Summarized, the LYRM containing domains LYRM6/ACPM- $\alpha$ and LYRM3/ACPM- $\beta$ play important roles in the activity and assembly/stability of complex I from yeast to human.

So far, only limited structural information is available about LYRMs. Prediction of secondary structures using the bioinformatics toolkit Quick2D (www.toolkit.tuebingen.mpg.de/quick2_d) suggested that they are small mainly $\alpha$-helical hydrophilic proteins. The molecular weight of mitochondrial complex I is almost $1 \mathrm{MDa}$ [27,37,38], and the LYRM6/ACPM domain (bovine B14/SDAP- $\alpha$ ) represents just $3 \%$ of the total protein mass [30,37]. The loss of this domain by chromosomal deletion of LYRM6 had no impact on the EPR signals of the (NADH reduced) Fe-S clusters of subcomplex LYRM6 $\Delta$, except for a minor effect on the signal intensity of Fe-S cluster N2 from the central subunit NDUFS7 (bovine PSST) [25]. Thus, the terminal Fe-S cluster N2, which is part of the ubiquinone reduction site, can be reduced and presents electrons to ubiquinone molecules. However, as 
mentioned above, subcomplex LYRM6 $\Delta$ is enzymatically inactive. Figure 2 shows the simplified structural models of mitochondrial complex I from bovine and Yarrowia lipolytica (Figure 2a,b, respectively). A tentative modeling of subunits LYRM6 and ACPM1 into the X-ray crystallographic map of Yarrowia lipolytica complex I (Figure 2c, green and magenta, respectively) provides structural insights into this domain [25]. Similar as reported for the bovine enzyme [24], LYRM6 is comprised of three $\alpha$-helices (Figure 2c, green) and interacts with the central subunits NDUFS3 and NDUFS2 (bovine $30 \mathrm{kDa}$ and $49 \mathrm{kDa}$ ) and on the distal side with ACPM1 (Figure 2c, magenta). Thus, ACPM1 contacts complex I exclusively via LYRM6 close to the ubiquinone reduction site. The structural analysis also suggests that the exposed negatively-charged phosphate moiety of the phosphopantetheine cofactor of ACPM1 (Figure 2c, yellow stick representation; see the red arrow) is critically involved in the binding to LYRM6. Interestingly, the contact site between LYRM6 and the central NDUFS2 subunit is a structural element that is critical for the catalytic activity of mitochondrial complex I [39]. The interaction of ACPM with this structural element might regulate complex I activity by so far unknown factors. Complex I subunit ACPM carries a long-chain acyl residue in its binding pocket $[34,37,40]$ that might function as one of these regulatory factors.

The duplicated interaction of respiratory complex I with ACPM seems to represent an essential protein-protein interaction that needs two adaptor LYRMs. Analyses of other LYRMs using the protein-protein interaction database STRING provides further insights into their functional protein association networks [41]. As expected, e.g., human LYRM4 interacts with the proteins of Fe-S cluster biogenesis, such as NFS1 and FXN, and complex V assembly factor FMC1 (LYR_2 motif) from Saccharomyces cerevisiae forms a complex with the assembly factor ATP12 (Figure 3a,b). In addition, the STRING analysis shows that LYRM4 and FMC1 also interact with ACPM [42,43]. Accordingly, I suggest that the structure of the LYR motif might present a general adaptor for interaction with the ACPM protein. The exposed negatively-charged phosphate moiety of ACPM's cofactor might play an important role in the structural stabilization of the protein-protein interaction between ACPM and LYRMs(Figure 2c, [25]).

\subsection{Essential Factor of Fe-S Cluster Biogenesis (LYRM4)}

The biosynthesis of Fe-S clusters in the matrix of mitochondria is an essential pathway of eukaryotic cells $[17,20,44]$. LYRM4 (ISD11) has been shown to interact with the ancient Fe-S cluster biogenesis components NFS1 (cysteine desulfurase), the scaffold protein ISCU and frataxin (FXN, Friedreich ataxia protein) $[16,45,46]$. The LYRM4/NFS1/FXN complex has been suggested to provide the Fe and $\mathrm{S}$ atoms for the Fe-S cluster assembly occurring at the ISCU scaffold proteins. The transfer of Fe-S clusters to the target proteins is performed by the ISCU/HSPA9/HSC20 complex [20]. Depletion of LYRM4 by siRNA in human HEK293T cells resulted in an increased intracellular Fe content, as is observed in Friedreich's ataxia, caused by defects in FXN [45]. Mutations of the L-Y-R sequence in LYRM4 from Saccharomyces cerevisiae resulted in partial loss of Nfs1 function [47]. Recently, LYRM4 was shown to influence the oligomeric state of NFS1 [48]. Interestingly, specific interactions of the lipid phosphatidylinositol 3,5-biphosphate (PIP2, substrate of PI3K) with LYRM4 were shown in profiles of lipid-protein interactions from Saccharomyces cerevisiae [49]. 
The mitochondrial production of $\mathrm{Fe}-\mathrm{S}$ clusters is essential to generate $\mathrm{Fe}-\mathrm{S}$ cluster-containing proteins. Furthermore, the availability of 4Fe-4S clusters (sulfur donors) and octanoyl-ACPM are both essential for the production of lipoic acid. Lipoic acid synthase (LIAS) exploits 4Fe-4S clusters to transfer two sulfur atoms to octanoyl-loaded target proteins, forming the lipoylated protein products [50,51]. In this context the protein-protein interaction of (octanoyl-)ACPM with the NFS1/LYRM4 complex might make sense (Figure 3a).
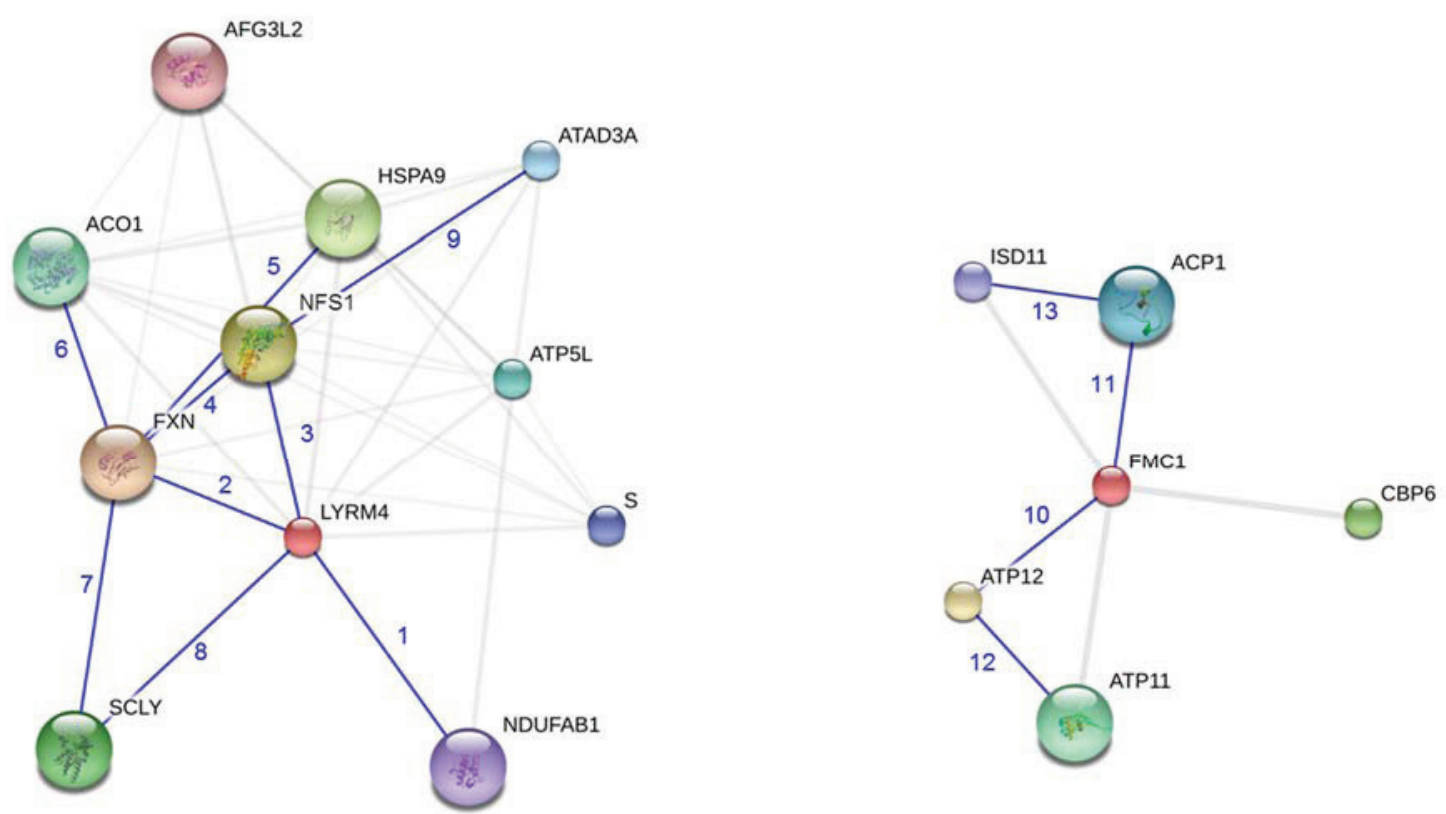

\section{a}

\section{b}

Figure 3. Graphical description of protein-protein interactions of human LYRM4 and FMC1 from $S$. cerevisiae using the bioinformatics tool STRING (modified actions views; [41]). (a) LYRM4 (red colored sphere) interacts with the enzymes of Fe-S cluster biogenesis, NFS1 and FXN. In addition, the interactions with selenocysteine lyase (SCLY) and ACPM (NDUFAB1) are shown. (b) FMC1 (red colored sphere) interacts with complex V assembly factor ATP12 and with ACPM (ACP1). Blue numbers indicate the experimentally shown protein-protein interactions (relevant information transferred from other species): 1, LYRM4-NDUFAB1 [43]; 2, LYRM4-FXN [45]; 3, LYRM4-NFS1 [43]; 4, FXN-NFS1 [52]; 5, FXN-HSPA9 (heat shock 70-kDa protein 9) [45]; 6, FXN-ACO1 (aconitase 1) [53,54]; 7, FXN-SCLY (by homology to NFS1); 8, LYRM4-SCLY (by homology to NFS1); 9, NFS1-ATAD3A (ATPase, AAA containing domain 3A) [43]; 10, FMC1-ATP12 [42]; 11, FMC1-ACP1 [42]; 12, ATP12-ATP11 [42]; 13, ACP1-ISD11 [42]. AFG3L2, AFG3 ATPase family gene 3-like 2; ATP5L, ATP synthase subunit G; S, cDNA FLJ78714; CBP6, cytochrome B pre-mRNA-processing protein 6.

\subsection{Complex I-Related LYRM5}

LYRM5 is a complex 1 phylogenetic profile (COPP) protein [3]. COPPs are absent in organisms that do not contain respiratory complex I. Accordingly, many complex I assembly factors are COPPs [55]. 
Enzymes involved in the metabolism of branched-chain amino acids (BCAA) and branched-chain fatty acids (BCFA) are also COPPs. BCAA and lipids were suggested to play a central role in the development of insulin resistance in diabetes [56]. Leucine regulates the activation of the mammalian target of rapamycin (mTOR) complex 1, which results in uncoupling of insulin signaling [57]. In Caenorhabditis elegans, a specific BCFA is involved in the post-embryonic growth control in parallel with the insulin receptor signaling pathway [58,59]. Furthermore, a BCFA induces mitochondrial-mediated apoptosis in human bladder cancer cells by regulation of the Akt/PKB and MAPK phosphorylation pathways [60]. Interestingly, the mitochondrial metabolism of BCAA by the branched chain $\alpha$-keto dehydrogenase (cofactor lipoic acid) and the biosynthesis of organelle-specific lipids (fatty acids) both depend on the biosynthetic function of complex I subunit ACPM/NDUFAB1 that produces mitochondrial acyl chains de novo [61].

\subsection{Assembly Factors of OXPHOS Complexes (LYRM7, LYRM8, ACN9, FMC1)}

The assembly of succinate:ubiquinone oxidoreductase (SDH, complex II) subunit SDHB, which contains three Fe-S clusters [62], is mediated by the two LYRM assembly factors, LYRM8/SDHAF1 and ACN9/SDHAF3 [5,18]. The LYRMs assist the maturation of SDHB and probably protect the transferred Fe-S clusters from oxidative stress [5]. Both LYRMs improve the production of complex II and increase mitochondrial complex II abundance. Accordingly, pathogenic mutations in LYRM8 induce SDH-defective infantile leukoencephalopathy [14]. Both LYRMs do not take part in the direct insertion of the Fe-S clusters into subunit SDHB. However, the binding of HSC20 to SDHB and LYRM8 guides Fe-S cluster delivery and likely assists Fe-S cluster insertion [18,20]. LYRM8/SDHAF1 and ACN9/SDHAF3 were suggested to protect SDHB from oxidative stress during the assembly process [5]. High concentrations of reactive oxygen species (ROS) damage Fe-S clusters, and protection of the sensitive Fe-S clusters is important in the presence of oxidative stress. Complex I and III produce a significant amount of ROS, and as shown recently, complex II is also discussed to play a role in mitochondrial ROS generation [63].

The dimer of ubiquinol:cytochrome $c$ oxidoreductase ( $b c 1$ complex, complex III) contains two Fe-S cluster-containing Rieske proteins (UQCRFS1) [64]. LYRM7 has been described as an accessory factor that stabilizes the Rieske protein prior to assembly to complex III without a chaperone-like function for insertion of the Fe-S cluster [65,66]. Recently, the same function was shown for the human LYRM7/MZM1L protein [67]. Mutation of the tyrosine in the L-Y-R sequence of complex III assembly factor LYRM7/Mzm1 from Saccharomyces cerevisiae resulted in the decrease of functional complex III [65], and human pathogenic mutation D25N in LYRM7/MZM1L induces the severe reduction of complex III activity [13]. Similar to LYRM8 and SDHB, LYRM7 interacts with the co-chaperone HSC20 [18]. This protein-protein interaction might protect the Rieske subunit from oxidative stress and support the stability until the protein is inserted into the membrane.

Human formation of mitochondrial complexes protein 1 (FMC1, Table 1) is not recognized as LYRM by the Pfam search tool; however, other mammalian and yeast homologs are members of the LYR_2 family. Mitochondrial ATP synthase (complex V) assembly factor FMC1 interacts with ATP12 [42], which plays a central role in the assembly of the $\mathrm{F}_{1}$ component of ATP synthase [6] (Figure 3b). 


\subsection{LYRM1, LYRM2 and LYRM9}

The gene of LYRM1 is highly expressed in omental adipose tissue of obese individuals and is an obesity-related gene involved in adipocyte differentiation and growth $[7,68,69]$. The overexpression of LYRM1 in mature 3T3-L1 adipocytes resulted in reduced insulin-stimulated uptake of glucose and impaired mitochondrial function [70]. High ROS levels were observed that were suggested to trigger the development of mitochondrial dysfunction and insulin-resistance. The knock-down of LYRM1 in these cells resulted in improved mitochondrial function [9] and even rescued the destructive effects of the uncoupling compound, carbonyl cyanide-4-(trifluoromethoxy)-phenylhydrazone (FCCP) [71]. Interestingly, the supply of lipoic acid had a positive effect on insulin-dependent signaling in L6 myotubes [72] and ameliorated the negative effects induced by LYRM1 overexpression [73]. A similar outcome was observed for the anti-diabetic drug, Metformin [74], which is also an inhibitor of respiratory complex I [75]. Overall, these results suggest that LYRM1 takes part in a metabolic redox-dependent signaling pathway connected to mitochondrial biogenesis and homeostasis (PI3K, Akt/PKB pathway).

LYRM2 is the least understood LYRM. There are indications that this protein of higher eukaryotes is involved in DNA repair [76].

The LYRM9 gene (C17orf108) has been identified as one of four response biomarkers for the immunosuppressive compound, MPA (mycophenolic acid), which is commonly used to treat patients with solid organ transplants [77]. LYRM9 was also discussed in the context of eosinophilic airway inflammation and particularly in association with childhood asthma [78]. The fraction of exhaled nitric oxide values (Feno) was related to specific asthma phenotypes. Single-nucleotide polymorphisms (SNP) in the genes of inducible NO synthase NOS2 and LYRM9 were suggested to affect Feno values.

\section{Multi-Domain Organization of LYRMs}

Supplementary information about the function of LYRMs might be provided by the analysis of the Pfam domain organization of several predicted, though unreviewed, proteins from the UniProt database [1]. The domain organization of the Pfam clan LYR-like CL0491 predicts 30 LYRMs in protein fusions with other protein domains of different function (http://pfam.xfam.org/clan/LYR-like\#tabview=tab1). Many predictions of multi-domain proteins might be wrong simply by errors in protein annotation; however, some constructs might be physiologically reasonable, e.g., the fusion of an LYRM assembly factor with another mitochondrial assembly factor. However, in Saccharomyces pombe, it has been demonstrated that the mitochondrial protein, tandem Rsm22-Cox11, does not function as a fused multi-domain protein [79]. In fact, after the import of this polypeptide into mitochondria, the protein domains are cleaved, resulting in matured individual proteins. Nevertheless, Table 2 shows nine selected candidates for fused LYRMs that might occur in the respective organisms. In Penicillium marneffei and Talaromyces stipitatus, complex II assembly factor LYRM8 is fused to HSP20 in an annotated multi-domain protein (UniProt entries B6QRH6_PENMQ and B8M7E8_TALSN). The protein-protein interaction of LYRM8 and the co-chaperone HSC20 has already been demonstrated [18]. HSP20 is a different chaperone; however, the combination of LYRM8 with a heat shock protein might support its chaperone-like function. The same is possibly true for complex III assembly factor LYRM7, which is fused to the 
HSP20-like HSP90 binding module (CS domain) in the slime mold, Dictyostelium fasciculatum (UniProt Entry F4Q9B8_DICFS). In the Chinese hamster Cricetulus griseus, the annotated gene sequence of LYRM9 is fused to the gene of inducible NO synthase (NOS2) (UniProt Entry G3GTQ5_CRIGR). The common functional background of human LYRM9 and NOS2 has been studied in genetic analyses of SNPs in the context of airway inflammation [78]. In addition, complex I assembly factor IND1/NUBPL has been suggested to play a role in the mitochondrial translation process [80-82]. The fusion of complex II assembly factor ACN9/SDHAF3 with a ribosomal processing protein (RRP7) in the yeast Pichia pastoris (UniProt Entry F2QP71_PICP7) might reflect a similar situation for complex II. LYRM1 is discussed to represent a factor in the insulin signaling pathway. In Salpingoeca sp., LYRM1 is fused to the mitochondrial small GTPase RAB8 (UniProt Entry F2USF2_SALS5). The fusion of LYRM1 with a Ras-like protein seems to present an interesting overlap of signal transduction pathways. Furthermore, FCM1 is fused to a RAP guanine nucleotide exchange factor (RAP-GEF) in three fungi (UniProt Entries B8MGM3_TALSN, Q5BG81_EMENI and A2Q880_ASPNC). In these organisms, the assembly of the F1 fragment from complex V might be connected to the RAP signaling pathway. In five insect species, LYRM4 is fused to the mitochondrial translational releasing factor RF1 (UniProt Entries B4GAC8_DROPE, B4MJX9_DROWI, Q291R9_DROPS, E3XFW0_ANODA and E9IC99_SOLIN). Thus, the biogenesis of $\mathrm{Fe}-\mathrm{S}$ clusters might be directly coupled to the production of the mitochondrially-encoded subunits of OXPHOS complexes. Overall, regarding the finding that mitochondrial protein tandems are cleaved from each other, the fusion of LYRMs to other protein domains might at least increase the import efficiency of the LYRM constructs and/or coordinate the expression levels of the fused domains, as suggested for Cox11 and Rsm22 in S. pombe [79].

\section{Conclusions}

Nuclear-encoded LYRMs of eukaryotes are primarily mitochondrial proteins. In many cases, they function as mitochondrial adaptor proteins and are accessory factors (LYR factors) controlling mitochondrial homeostasis. Mitochondria are the central organelles for efficient ATP production and also play important roles in the induction of apoptosis, $\mathrm{Ca}^{2+}$ homeostasis, regulation of cell cycle and Fe-S cluster biogenesis. The assembly and full functionality of mitochondrial OXPHOS complexes depends on intact LYRMs. It is tempting to speculate that the specialization of mitochondria required new factors for the efficient assembly of OXPHOS complexes. Furthermore, mitochondrial oxidative stress might have required protective mechanisms, since Fe-S clusters are sensitive to ROS. LYRM8, which interacts with the co-chaperone HSC20 of the ISCU/HSPA9 complex, and ACN9/SDHAF3 protect Fe-S clusters during the transfer to complex II subunit SDHB. Four LYRMs interact with ACPM, which is the central component of the mitochondrial fatty acid synthesis machinery that produces octanoyl-ACPM precursors for the essential endogenous biosynthesis of lipoic acid. The physiological role of the LYRM/ACPM interaction is still unknown; however, the adaptor-like function of LYRMs to bind ACPM is suggested in this review. Overall, LYRMs play multifaceted roles in bioenergetic, biosynthetic and metabolic signaling pathways that promote mitochondrial biogenesis. 
Table 2. Selection of LYRMs in domain organization with other proteins. Please note that all proteins are unreviewed, predicted proteins of the UniProt database analyzed by the Pfam data base.

\begin{tabular}{|c|c|c|c|c|c|c|}
\hline LYRM a & Fused Domain & Organism(s) & Source & $\begin{array}{c}\text { LYRM Domain, } \\
\text { Amino Acid Residue } \\
\text { (Start-End) }\end{array}$ & $\begin{array}{c}\text { Human } \\
\text { Orthologue }\end{array}$ & Pfam Domain, Remarks \\
\hline LYRM1 & Ras & Salpingoeca rosetta & http://pfam.xfam.org/protein/F2USF2_SALS5 & $180-230$ & RAB8B_HUMAN ${ }^{b}$ & $\begin{array}{l}\text { PF00071, small GTP } \\
\text { binding protein RAB8 }\end{array}$ \\
\hline LYRM2 & BTB-SPRY & Naegleria gruberi & http://pfam.xfam.org/protein/D2VKV5_NAEGR & $462-524$ & KBTB3_HUMAN & $\begin{array}{l}\text { PF00651/PF00622, } \\
\text { BTB/POZ protein }\end{array}$ \\
\hline LYRM3 & DEAD/DEAH & $\begin{array}{c}\text { Selaginella } \\
\text { moellendorffii }\end{array}$ & http://pfam.xfam.org/protein/D8TAY1_SELML & $22-81$ & DDX28_HUMAN b & $\begin{array}{c}\text { PF00270, RNA helicase, } \\
\text { RNA processing }\end{array}$ \\
\hline LYRM4 & RF-1 & $\begin{array}{l}\text { Drosophila } \\
\text { persimili }^{\mathrm{c}}\end{array}$ & http://pfam.xfam.org/protein/B4GAC8_DROPE & $7-61$ & CL065_HUMAN ${ }^{\mathrm{b}}$ & $\begin{array}{l}\text { PF00472, translational } \\
\text { release factor } 1, \\
\text { C12orf65 }\end{array}$ \\
\hline LYRM7 & CS (HSP20-like) & $\begin{array}{l}\text { Dictyostelium } \\
\text { fasciculatum }\end{array}$ & http://pfam.xfam.org/protein/F4Q9B8_DICFS & $6-68$ & - & $\begin{array}{l}\text { PF04969, binding } \\
\text { module for HSP90 }\end{array}$ \\
\hline LYRM8 & HSP20 & $\begin{array}{l}\text { Penicillium } \\
\text { marneffei }^{\mathrm{d}}\end{array}$ & http://pfam.xfam.org/protein/B6QRH6_PENMQ & $105-164$ & CRYAA_HUMAN & PF00011 \\
\hline LYRM9 & NO_synthase & Cricetulus griseus & http://pfam.xfam.org/protein/G3GTQ5_CRIGR & $11-69$ & NOS2_HUMAN & $\begin{array}{l}\text { PF02898, inducible } \\
\text { NO synthase }\end{array}$ \\
\hline ACN9 & RRP7 & Pichia pastoris & http://pfam.xfam.org/protein/F2QP71_PICP7 & $10-67$ & RRP7A_HUMAN & $\begin{array}{c}\text { PF12923, ribosomal } \\
\text { RNA-processing protein } 7\end{array}$ \\
\hline FMC1 & Ras-GEF & $\begin{array}{l}\text { Talaromyces } \\
\text { stipitatus }^{\mathrm{e}}\end{array}$ & http://pfam.xfam.org/protein/B8MGM3_TALSN & $568-659$ & RPGF3_HUMAN & $\begin{array}{c}\text { PF00617, RAP guanine } \\
\text { nucleotide exchange } \\
\text { factor } 3\end{array}$ \\
\hline
\end{tabular}

${ }^{a}$ The identity of the LYRM domains was deduced using the bioinformatics tool, Standard Protein BLAST, with default parameters [83]; ${ }^{\mathrm{b}}$ mitochondrial protein; ${ }^{\mathrm{c}}$ also in Drosophila willistoni B4MJX9_DROWI, Drosophila pseudoobscura Q291R9_DROPS, Anopheles darling E3XFW0_ANODA, Solenopsis invicta E9IC99_SOLIN; d also in Talaromyces stipitatus B8M7E8_TALSN; ${ }^{\mathrm{e}}$ also in Aspergillus niger Q5BG81_EMENI, Emericella nidulans A2Q880_ASPNC. 


\section{Acknowledgments}

I would like to thank Volker Zickermann for support and for critically reading the manuscript before submission.

\section{Conflicts of Interest}

The authors declare no conflict of interest

\section{References}

1. Finn, R.D.; Bateman, A.; Clements, J.; Coggill, P.; Eberhardt, R.Y.; Eddy, S.R.; Heger, A.; Hetherington, K.; Holm, L.; Mistry, J.; et al. Pfam: The protein families database. Nucleic Acids Res. 2014, 42, D222-D230.

2. Angerer, H. The superfamily of mitochondrial Complex1_LYR motif-containing (LYRM) proteins. Biochem. Soc. Trans. 2013, 41, 1335-1341.

3. Pagliarini, D.J.; Calvo, S.E.; Chang, B.; Sheth, S.A.; Vafai, S.B.; Ong, S.E.; Walford, G.A.; Sugiana, C.; Boneh, A.; Chen, W.K.; et al. A mitochondrial protein compendium elucidates complex I disease biology. Cell 2008, 134, 112-123.

4. Dennis, R.A.; McCammon, M.T. ACN9 is a novel protein of gluconeogenesis that is located in the mitochondrial intermembrane space. Eur. J. Biochem. 1999, 261, 236-243.

5. Na, U.; Yu, W.; Cox, J.; Bricker, D.K.; Brockmann, K.; Rutter, J.; Thummel, C.S.; Winge, D.R. The LYR factors SDHAF1 and SDHAF3 mediate maturation of the iron-sulfur subunit of succinate dehydrogenase. Cell Metab. 2014, 20, 253-266.

6. Lefebvre-Legendre, L.; Vaillier, J.; Benabdelhak, H.; Velours, J.; Slonimski, P.P.; di Rago, J.P. Identification of a nuclear gene (FMC1) required for the assembly/stability of yeast mitochondrial F(1)-ATPase in heat stress conditions. J. Biol. Chem. 2001, 276, 6789-6796.

7. Qiu, J.; Gao, C.L.; Zhang, M.; Chen, R.H.; Chi, X.; Liu, F.; Zhang, C.M.; Ji, C.B.; Chen, X.H.; Zhao, Y.P.; et al. LYRM1, a novel gene promotes proliferation and inhibits apoptosis of preadipocytes. Eur. J. Endocrinol. 2009, 160, 177-184.

8. Shi, Y.; Ghosh, M.C.; Tong, W.H.; Rouault, T.A. Human ISD11 is essential for both iron-sulfur cluster assembly and maintenance of normal cellular iron homeostasis. Hum. Mol. Genet. 2009, 18, 3014-3025.

9. Zhu, G.Z.; Zhang, M.; Kou, C.Z.; Ni, Y.H.; Ji, C.B.; Cao, X.G.; Guo, X.R. Effects of LYRM1 knockdown on mitochondrial function in 3 T3-L1 murine adipocytes. J. Bioenerg. Biomembr. 2012, $44,225-232$.

10. Haack, T.B.; Madignier, F.; Herzer, M.; Lamantea, E.; Danhauser, K.; Invernizzi, F.; Koch, J.; Freitag, M.; Drost, R.; Hillier, I.; et al. Mutation screening of 75 candidate genes in 152 complex I deficiency cases identifies pathogenic variants in 16 genes including NDUFB9. J. Med. Genet. 2012, 49, 83-89. 
11. Lim, S.C.; Friemel, M.; Marum, J.E.; Tucker, E.J.; Bruno, D.L.; Riley, L.G.; Christodoulou, J.; Kirk, E.P.; Boneh, A.; DeGennaro, C.M.; et al. Mutations in LYRM4, encoding iron-sulfur cluster biogenesis factor ISD11, cause deficiency of multiple respiratory chain complexes. Hum. Mol. Genet. 2013, 22, 4460-4473.

12. Ladha, J.S.; Tripathy, M.K.; Mitra, D. Mitochondrial complex I activity is impaired during HIV-1-induced T-cell apoptosis. Cell Death. Differ. 2005, 12, 1417-1428.

13. Invernizzi, F.; Tigano, M.; Dallabona, C.; Donnini, C.; Ferrero, I.; Cremonte, M.; Ghezzi, D.; Lamperti, C.; Zeviani, M. A homozygous mutation in LYRM7/MZM1L associated with early onset encephalopathy, lactic acidosis, and severe reduction of mitochondrial complex III activity. Hum. Mutat. 2013, 34, 1619-1622.

14. Ghezzi, D.; Goffrini, P.; Uziel, G.; Horvath, R.; Klopstock, T.; Lochmuller, H.; D’Adamo, P.; Gasparini, P.; Strom, T.M.; Prokisch, H.; et al. SDHAF1, encoding a LYR complex-II specific assembly factor, is mutated in SDH-defective infantile leukoencephalopathy. Nat. Genet. 2009, 41, 654-656.

15. Dick, D.M.; Aliev, F.; Wang, J.C.; Saccone, S.; Hinrichs, A.; Bertelsen, S.; Budde, J.; Saccone, N.; Foroud, T.; Nurnberger, J., Jr.; et al. A Systematic single nucleotide polymorphism screen to fine-map alcohol dependence genes on chromosome 7 identifies association with a novel susceptibility gene ACN9. Biol. Psychiatry 2008, 63, 1047-1053.

16. Richards, T.A.; van der Giezen, M. Evolution of the Isd11-IscS complex reveals a single alpha-proteobacterial endosymbiosis for all eukaryotes. Mol. Biol. Evol. 2006, 23, 1341-1344.

17. Stehling, O.; Wilbrecht, C.; Lill, R. Mitochondrial iron-sulfur protein biogenesis and human disease. Biochimie 2014, 100, 61-77.

18. Maio, N.; Singh, A.; Uhrigshardt, H.; Saxena, N.; Tong, W.H.; Rouault, T.A. Cochaperone binding to LYR motifs confers specificity of iron sulfur cluster delivery. Cell Metab. 2014, 19, 445-457.

19. Cai, K.; Frederick, R.O.; Kim, J.H.; Reinen, N.M.; Tonelli, M.; Markley, J.L. Human mitochondrial chaperone (mtHSP70) and cysteine desulfurase (NFS1) bind preferentially to the disordered conformation, whereas co-chaperone (HSC20) binds to the structured conformation of the iron-sulfur cluster scaffold protein (ISCU). J. Biol. Chem. 2013, 288, 28755-28770.

20. Maio, N.; Rouault, T.A. Iron-sulfur cluster biogenesis in mammalian cells: New insights into the molecular mechanisms of cluster delivery. Biochim. Biophys. Acta 2014, doi:10.1016/j.bbamcr. 2014.09.009.

21. Claros, M.G.; Vincens, P. Computational method to predict mitochondrially imported proteins and their targeting sequences. Eur. J. Biochem. 1996, 241, 779-786.

22. Larkin, M.A.; Blackshields, G.; Brown, N.P.; Chenna, R.; McGettigan, P.A.; McWilliam, H.; Valentin, F.; Wallace, I.M.; Wilm, A.; Lopez, R.; et al. Clustal W and Clustal X version 2.0. Bioinformatics 2007, 23, 2947-2948.

23. Zickermann, V.; Wirth, C.; Nasiri, H.; Siegmund, K.; Schwalbe, H.; Hunte, C.; and Brandt, U. Structural biology. Mechanistic insight from the crystal structure of mitochondrial complex I. Science 2015, 347, 44-49.

24. Vinothkumar, K.R.; Zhu, J.; Hirst, J. Architecture of mammalian respiratory complex I. Nature 2014, 515, 80-84. 
25. Angerer, H.; Radermacher, M.; Mankowska, M.; Steger, M.; Zwicker, K.; Heide, H.; Wittig, I.; Brandt, U.; Zickermann, V. The LYR protein subunit NB4M/NDUFA6 of mitochondrial complex I anchors an acyl carrier protein and is essential for catalytic activity. Proc. Natl. Acad. Sci. USA 2014, 111, 5207-5212.

26. Hunte, C.; Zickermann, V.; Brandt, U. Functional modules and structural basis of conformational coupling in mitochondrial complex I. Science 2010, 329, 448-451.

27. Hirst, J. Mitochondrial complex I. Annu. Rev. Biochem. 2013, 82, 551-575.

28. Runswick, M.J.; Fearnley, I.M.; Skehel, J.M.; Walker, J.E. Presence of an acyl carrier protein in NADH:Ubiquinone oxidoreductase from bovine heart mitochondria. FEBS Lett. 1991, 286, 121-124.

29. Cronan, J.E.; Fearnley, I.M.; Walker, J.E. Mammalian mitochondria contain a soluble acyl carrier protein. FEBS Lett. 2005, 579, 4892-4896.

30. Angerer, H.; Zwicker, K.; Wumaier, Z.; Sokolova, L.; Heide, H.; Steger, M.; Kaiser, S.; Nubel, E.; Brutschy, B.; Radermacher, M.; et al. A scaffold of accessory subunits links the peripheral arm and the distal proton-pumping module of mitochondrial complex I. Biochem. J. 2011, 437, 279-288.

31. Dobrynin, K.; Abdrakhmanova, A.; Richers, S.; Hunte, C.; Kerscher, S.; Brandt, U. Characterization of two different acyl carrier proteins in complex I from Yarrowia lipolytica. Biochim. Biophys. Acta 2010, 1797, 152-159.

32. Feng, D.; Witkowski, A.; Smith, S. Down-regulation of mitochondrial acyl carrier protein in mammalian cells compromises protein lipoylation and respiratory complex I and results in cell death. J. Biol. Chem. 2009, 284, 11436-11445.

33. Hiltunen, J.K.; Autio, K.J.; Schonauer, M.S.; Kursu, V.A.; Dieckmann, C.L.; Kastaniotis, A.J. Mitochondrial fatty acid synthesis and respiration. Biochim. Biophys. Acta 2010, 1797, 1195-1202.

34. Schneider, R.; Massow, M.; Lisowsky, T.; Weiss, H. Different respiratory-defective phenotypes of Neurospora crassa and Saccharomyces cerevisiae after inactivation of the gene encoding the mitochondrial acyl carrier protein. Curr. Genet. 1995, 29, 10-17.

35. Goraca, A.; Huk-Kolega, H.; Piechota, A.; Kleniewska, P.; Ciejka, E.; Skibska, B. Lipoic acid-biological activity and therapeutic potential. Pharmacol. Rep. 2011, 63, 849-858.

36. Yi, X.; Maeda, N. Endogenous production of lipoic acid is essential for mouse development. Mol. Cell Biol. 2005, 25, 8387-8392.

37. Carroll, J.; Fearnley, I.M.; Shannon, R.J.; Hirst, J.; Walker, J.E. Analysis of the subunit composition of complex I from bovine heart mitochondria. Mol. Cell Proteomics. 2003, 2, 117-126.

38. Kmita, K.; Zickermann, V. Accessory subunits of mitochondrial complex I. Biochem. Soc. Trans. 2013, 41, 1272-1279.

39. Babot, M.; Birch, A.; Labarbuta, P.; Galkin, A. Characterisation of the active/de-active transition of mitochondrial complex I. Biochim. Biophys. Acta 2014, 1837, 1083-1092.

40. Brody, S.; Mikolajczyk, S. Neurospora mitochondria contain an acyl-carrier protein. Eur. J. Biochem. 1988, 173, 353-359.

41. Franceschini, A.; Szklarczyk, D.; Frankild, S.; Kuhn, M.; Simonovic, M.; Roth, A.; Lin, J.; Minguez, P.; Bork, P.; von, M.C.; et al. STRING v9.1: Protein-protein interaction networks, with increased coverage and integration. Nucleic Acids Res. 2013, 41, D808-D815. 
42. Krogan, N.J.; Cagney, G.; Yu, H.; Zhong, G.; Guo, X.; Ignatchenko, A.; Li, J.; Pu, S.; Datta, N.; Tikuisis, A.P.; et al. Global landscape of protein complexes in the yeast Saccharomyces cerevisiae. Nature 2006, 440, 637-643.

43. Guruharsha, K.G.; Rual, J.F.; Zhai, B.; Mintseris, J.; Vaidya, P.; Vaidya, N.; Beekman, C.; Wong, C.; Rhee, D.Y.; Cenaj, O.; et al. A protein complex network of Drosophila melanogaster. Cell 2011, 147, 690-703.

44. Lill, R.; Hoffmann, B.; Molik, S.; Pierik, A.J.; Rietzschel, N.; Stehling, O.; Uzarska, M.A.; Webert, H.; Wilbrecht, C.; Muhlenhoff, U. The role of mitochondria in cellular iron-sulfur protein biogenesis and iron metabolism. Biochim. Biophys. Acta 2012, 1823, 1491-1508.

45. Shan, Y.; Napoli, E.; Cortopassi, G. Mitochondrial frataxin interacts with ISD11 of the NFS1/ISCU complex and multiple mitochondrial chaperones. Hum. Mol. Genet. 2007, 16, 929-941.

46. Wiedemann, N.; Urzica, E.; Guiard, B.; Muller, H.; Lohaus, C.; Meyer, H.E.; Ryan, M.T.; Meisinger, C.; Muhlenhoff, U.; Lill, R.; et al. Essential role of Isd11 in mitochondrial iron-sulfur cluster synthesis on Isu scaffold proteins. EMBO J. 2006, 25, 184-195.

47. Pandey, A.; Golla, R.; Yoon, H.; Dancis, A.; Pain, D. Persulfide formation on mitochondrial cysteine desulfurase: Enzyme activation by a eukaryote-specific interacting protein and Fe-S cluster synthesis. Biochem. J. 2012, 448, 171-187.

48. Terali, K.; Beavil, R.L.; Pickersgill, R.W.; van der Giezen, M. The effect of the adaptor protein Isd1 1 on the quaternary structure of the eukaryotic cysteine desulphurase Nfs1. Biochem. Biophys. Res. Commun. 2013, 440, 235-240.

49. Lu, K.Y.; Tao, S.C.; Yang, T.C.; Ho, Y.H.; Lee, C.H.; Lin, C.C.; Juan, H.F.; Huang, H.C.; Yang, C.Y.; Chen, M.S.; et al. Profiling lipid-protein interactions using nonquenched fluorescent liposomal nanovesicles and proteome microarrays. Mol. Cell Proteomics. 2012, 11, 1177-1190.

50. Cicchillo, R.M.; Booker, S.J. Mechanistic investigations of lipoic acid biosynthesis in Escherichia coli: Both sulfur atoms in lipoic acid are contributed by the same lipoyl synthase polypeptide. J. Am. Chem. Soc. 2005, 127, 2860-2861.

51. Harmer, J.E.; Hiscox, M.J.; Dinis, P.C.; Fox, S.J.; Iliopoulos, A.; Hussey, J.E.; Sandy, J.; van Beek, F.T.; Essex, J.W.; Roach, P.L. Structures of lipoyl synthase reveal a compact active site for controlling sequential sulfur insertion reactions. Biochem. J. 2014, 464, 123-133.

52. Gerber, J.; Muhlenhoff, U.; Lill, R. An interaction between frataxin and Isu1/NFS1 that is crucial for $\mathrm{Fe} / \mathrm{S}$ cluster synthesis on Isu1. EMBO Rep. 2003, 4, 906-911.

53. Condo, I.; Malisan, F.; Guccini, I.; Serio, D.; Rufini, A.; Testi, R. Molecular control of the cytosolic aconitase/IRP1 switch by extramitochondrial frataxin. Hum. Mol. Genet. 2010, 19, 1221-1229.

54. Bulteau, A.L.; O’Neill, H.A.; Kennedy, M.C.; Ikeda-Saito, M.; Isaya, G.; Szweda, L.I. Frataxin acts as an iron chaperone protein to modulate mitochondrial aconitase activity. Science 2004, 305, 242-245.

55. Mimaki, M.; Wang, X.; McKenzie, M.; Thorburn, D.R.; Ryan, M.T. Understanding mitochondrial complex I assembly in health and disease. Biochim. Biophys. Acta 2012, 1817, 851-862.

56. Newgard, C.B. Interplay between lipids and branched-chain amino acids in development of insulin resistance. Cell Metab. 2012, 15, 606-614.

57. Lynch, C.J.; Adams, S.H. Branched-chain amino acids in metabolic signalling and insulin resistance. Nat. Rev. Endocrinol. 2014, 10, 723-736. 
58. Kniazeva, M.; Euler, T.; Han, M. A branched-chain fatty acid is involved in post-embryonic growth control in parallel to the insulin receptor pathway and its biosynthesis is feedback-regulated in C. elegans. Genes Dev. 2008, 22, 2102-2110.

59. Kniazeva, M.; Shen, H.; Euler, T.; Wang, C.; Han, M. Regulation of maternal phospholipid composition and IP(3)-dependent embryonic membrane dynamics by a specific fatty acid metabolic event in C. elegans. Genes Dev. 2012, 26, 554-566.

60. Lin, T.; Yin, X.; Cai, Q.; Fan, X.; Xu, K.; Huang, L.; Luo, J.; Zheng, J.; Huang, J. 13-Methyltetradecanoic acid induces mitochondrial-mediated apoptosis in human bladder cancer cells. Urol. Oncol. Semin. Orig. Investig. 2012, 30, 339-345.

61. Zensen, R.; Husmann, H.; Schneider, R.; Peine, T.; Weiss, H. De novo synthesis and desaturation of fatty acids at the mitochondrial acyl-carrier protein, a subunit of NADH:Ubiquinone oxidoreductase in Neurospora crassa. FEBS Lett. 1992, 310, 179-181.

62. Lancaster, C.R.; Kroger, A. Succinate: Quinone oxidoreductases: New insights from X-ray crystal structures. Biochim. Biophys. Acta 2000, 1459, 422-431.

63. Drose, S.; Brandt, U. Molecular mechanisms of superoxide production by the mitochondrial respiratory chain. Adv. Exp. Med. Biol. 2012, 748, 145-169.

64. Crofts, A.R. The cytochrome bc1 complex: Function in the context of structure. Annu. Rev. Physiol. 2004, 66, 689-733.

65. Atkinson, A.; Smith, P.; Fox, J.L.; Cui, T.Z.; Khalimonchuk, O.; Winge, D.R. The LYR protein Mzm1 functions in the insertion of the Rieske Fe/S protein in yeast mitochondria. Mol. Cell Biol. 2011, 31, 3988-3996.

66. Cui, T.Z.; Smith, P.M.; Fox, J.L.; Khalimonchuk, O.; Winge, D.R. Late-stage maturation of the Rieske Fe/S protein: Mzm1 stabilizes Rip1 but does not facilitate its translocation by the AAA ATPase Bcs1. Mol. Cell Biol. 2012, 32, 4400-4409.

67. Sanchez, E.; Lobo, T.; Fox, J.L.; Zeviani, M.; Winge, D.R.; Fernandez-Vizarra, E. LYRM7/MZM1L is a UQCRFS1 chaperone involved in the last steps of mitochondrial complex III assembly in human cells. Biochim. Biophys. Acta 2013, 1827, 285-293.

68. Zhu, S.; Cheng, G.; Zhu, H.; Guan, G. A study of genes involved in adipocyte differentiation. J. Pediatr. Endocrinol. Metab 2014,

69. Yin, C.; Xiao, Y.; Zhang, W.; Xu, E.; Liu, W.; Yi, X.; Chang, M. DNA microarray analysis of genes differentially expressed in adipocyte differentiation. J. Biosci. 2014, 39, 415-423.

70. Cao, X.G.; Kou, C.Z.; Zhao, Y.P.; Gao, C.L.; Zhu, C.; Zhang, C.M.; Ji, C.B.; Qin, D.N.; Zhang, M.; Guo, X.R. Overexpression of LYRM1 induces mitochondrial impairment in 3T3-L1 adipocytes. Mol. Genet. Metab. 2010, 101, 395-399.

71. Zhang, M.; Qin, Z.Y.; Dai, Y.M.; Wang, Y.M.; Zhu, G.Z.; Zhao, Y.P.; Ji, C.B.; Zhu, J.G.; Shi, C.M.; Qiu, J.; et al. Knockdown of LYRM1 rescues insulin resistance and mitochondrial dysfunction induced by FCCP in 3T3-L1 adipocytes. Cell Biochem. Biophys. 2014, 70, 667-675.

72. Kou, C.; Cao, X.; Qin, D.; Ji, C.; Zhu, J.; Zhang, C.; Zhu, C.; Gao, C.; Chen, R.; Guo, X.; et al. Over-expression of LYRM1 inhibits glucose transport in rat skeletal muscles via attenuated phosphorylation of PI3K (p85) and Akt. Mol. Cell Biochem. 2011, 348, 149-154. 
73. Qin, Z.Y.; Zhang, M.; Guo, X.R.; Wang, Y.M.; Zhu, G.Z.; Ni, Y.H.; Zhao, Y.P.; Qiu, J.; Kou, C.Z.; Qin, R.; et al. Alpha-lipoic acid ameliorates impaired glucose uptake in LYRM1 overexpressing 3T3-L1 adipocytes through the IRS-1/Akt signaling pathway. J. Bioenerg. Biomembr. 2012, 44, 579-586.

74. Qin, Z.Y.; Zhang, M.; Dai, Y.M.; Wang, Y.M.; Zhu, G.Z.; Zhao, Y.P.; Ji, C.B.; Qiu, J.; Cao, X.G.; Guo, X.R. Metformin prevents LYRM1-induced insulin resistance in 3T3-L1 adipocytes via a mitochondrial-dependent mechanism. Exp. Biol. Med. (Maywood.) 2014, 239, 1567-1574.

75. Bridges, H.R.; Jones, A.J.; Pollak, M.N.; Hirst, J. Effects of metformin and other biguanides on oxidative phosphorylation in mitochondria. Biochem. J. 2014, 462, 475-487.

76. Lu, X.; Shao, J.; Li, H.; Yu, Y. Temporal gene expression changes induced by a low concentration of benzo[a]pyrene diol epoxide in a normal human cell line. Mutat. Res. 2010, 684, 74-80.

77. Wu, T.Y.; Fridley, B.L.; Jenkins, G.D.; Batzler, A.; Wang, L.; Weinshilboum, R.M. Mycophenolic acid response biomarkers: A cell line model system-based genome-wide screen. Int. Immunopharmacol. 2011, 11, 1057-1064.

78. Van der Valk, R.J.; Duijts, L.; Timpson, N.J.; Salam, M.T.; Standl, M.; Curtin, J.A.; Genuneit, J.; Kerhof, M.; Kreiner-Moller, E.; Caceres, A.; et al. Fraction of exhaled nitric oxide values in childhood are associated with 17q11.2-q12 and 17q12-q21 variants. J. Allergy Clin. Immunol. 2014, 134, 46-55.

79. Khalimonchuk, O.; Ott, M.; Funes, S.; Ostermann, K.; Rodel, G.; Herrmann, J.M. Sequential processing of a mitochondrial tandem protein: Insights into protein import in Schizosaccharomyces pombe. Eukaryot. Cell 2006, 5, 997-1006.

80. Chakrabarti, V.S.; Mikolajczyk, M.; Boscaro, F.; Calderone, V. Human Ind1 expression causes over-expression of E. coli beta-lactamase ampicillin resistance protein. Protein Expr. Purif. 2014, $104 C, 26-33$.

81. Wydro, M.M.; Sharma, P.; Foster, J.M.; Bych, K.; Meyer, E.H.; Balk, J. The evolutionarily conserved iron-sulfur protein INDH is required for complex I assembly and mitochondrial translation in Arabidopsis (corrected). Plant Cell 2013, 25, 4014-4027.

82. Calvo, S.E.; Tucker, E.J.; Compton, A.G.; Kirby, D.M.; Crawford, G.; Burtt, N.P.; Rivas, M.; Guiducci, C.; Bruno, D.L.; Goldberger, O.A.; et al. High-throughput, pooled sequencing identifies mutations in NUBPL and FOXRED1 in human complex I deficiency. Nat. Genet. 2010, 42, 851-858.

83. Boratyn, G.M.; Camacho, C.; Cooper, P.S.; Coulouris, G.; Fong, A.; Ma, N.; Madden, T.L.; Matten, W.T.; McGinnis, S.D.; Merezhuk, Y.; et al. BLAST: A more efficient report with usability improvements. Nucleic Acids Res. 2013, 41, W29-W33.

(C) 2015 by the authors; licensee MDPI, Basel, Switzerland. This article is an open access article distributed under the terms and conditions of the Creative Commons Attribution license (http://creativecommons.org/licenses/by/4.0/). 